GEOPHYSICAL RESEARCH LETTERS, VOL. 13, NO. 3, PAGES 299-301, MARCH 1986

\title{
MODELING OF DUST HALO FORMATION FOLLOWING COMET OUTBURSTS: PRELIMINARY RESULTS
}

\section{T.I. Gombosi*}

Space Physics Research Laboratory,The University of Michigan, Ann Arbor, MI 48109

\author{
M. Horányi*
}

Supercomputer Computations Research Institute, Florida State University

Tallahassee, FL 32306

Abstract Evolution of gas and dust distributions following a spatially and temporally localized comet outburst was calculated using a hybrid kinetic - hydrodynamic method. It was found that a comet outburst resulted in significantly increased dust terminal velocities. As these higher terminal velocity values result in larger apex distances, therefore the outburst generates distinct dust envelopes in front of the regular dust coma. Such envelopes were observed at several comets (cf. comet Donati).

\section{Introduction}

It was recognized as early as the mid 1930 s that gas outflow plays an important role in the cometary dust production process (Orlov [1935]). Interacting two component (gas and dust) model of released cometary material were published almost simultaneously by Probstein [1968], Brunner and Michel [1968] and Shulman [1969]. A large number of papers dealing with various aspects of cometary flows have since been published (for recent reviews see Mendis et al. [1985], Gombosi et al. [1986]). Over the last fifteen years a variety of approaches have been adopted, including hydrodynamic, dusty hydrodynamic and kinetic models. However, even though both the formulation of the governing equations and the methods of solution have been improved significantly since the original works, until recently comet coma models have been based on steady-state calculations. Recently Gombosi et al. [1985] and Kitamura [1986] have published the first time varying dusty gas dynamic calculations of the inner coma region, where the gas-dust interaction takes place.

The main goal of this paper is to assess the role of inner coma gas-dust interaction on outer coma dust halo formation and evolution. In order to achieve this goal we combined the dusty gas dynamic inner coma model of Gombosi et al. [1985] with the kinetic dust model of Horanyi and Mendis [1985] which calculates individual dust grain trajectories at larger cometocentric distances. The original method of Gombosi et al. [1985] was extended to include a realistic dust size distribution (with 12 dust sizes) and gas heating due to infrared radiation trapping (Marconi and Mendis [1986]). This type of hybrid calculation enabled us to calculate both the small and large scale temporal and spatial evolutions of gas and dust distributions following a spatially and temporally localized comet outburst.

\section{Model}

In the present model a slowly rotating ( $T>>2$ hours), dark nucleus (bolometric albedo $=0.05$ ) composed of water ice and

\footnotetext{
*also at Central Research Institute for Physics, Hungarian Academy of Sciences, Budapest, Hungary
}

Copyright 1986 by the American Geophysical Union. non-volatile dust was considered at a heliocentric distance of $0.83 \mathrm{AU}$ from the sun (corresponding to Comet Halley's position on March 9, 1986 (VEGA-2 encounter date)). The nucleus had a radius of $3 \mathrm{~km}$, and initially had a $200 \mathrm{~K}$ isothermal surface temperature, resulting in uniform gas and dust production rates of $1.1 \times 10^{-5} \mathrm{~g} / \mathrm{cm}^{2} / \mathrm{sec}$ and $2.9 \times 10^{-6}$ $\mathrm{g} / \mathrm{cm}^{2} / \mathrm{sec}$, respectively. At a time $\mathrm{t}=0$ a 2 hours long, localized (angular extension $=30^{\circ}$ ), subsolar surface temperature jump (to $210 \mathrm{~K}$ ) generated a comet outburst, resulting in approximately four times larger gas and dust production rates from this area. Such a sudden increase can be caused by several possible mechanisms (such as surface inhomogenities).Because the evaporation rate is proportional to $\exp \left(-\right.$ const $\left./ \mathrm{T}_{\text {surf }}\right)$ a small change in the surface temperature can generate a large outburst.

In the inner coma $(r<300 \mathrm{~km})$, where the gas drag accelerates the dust particles the time-dependent, spherically symmetric gas continuity, momentum, and energy equations were solved (cf. Gombosi et al. [1985]).

The momentum and energy tranfer rates from the gas to the dust component can be obtained by simultaneously solving the momentum and energy balance equations for the different size dust particles (12 logarithmically spaced dust sizes were employed between 0.1 and $100 \mu \mathrm{m}$ ) with the gas equations, and in each step calculating the transfer functions. The details of such a method were originally published by Probstein [1968]. In the present calculation a modified version of the original Probstein technique as described by Gombosi et al. [1985] was adopted. When determining the external heat source rate we tried to retain simplicity as much as possible, yet include the most important processes. The main contribution to the photochemical heating of an $\mathrm{H}_{2} \mathrm{O}$ dominated inner cometary atmosphere comes from the photodissociation of water molecules (cf. Huebner [1985]).

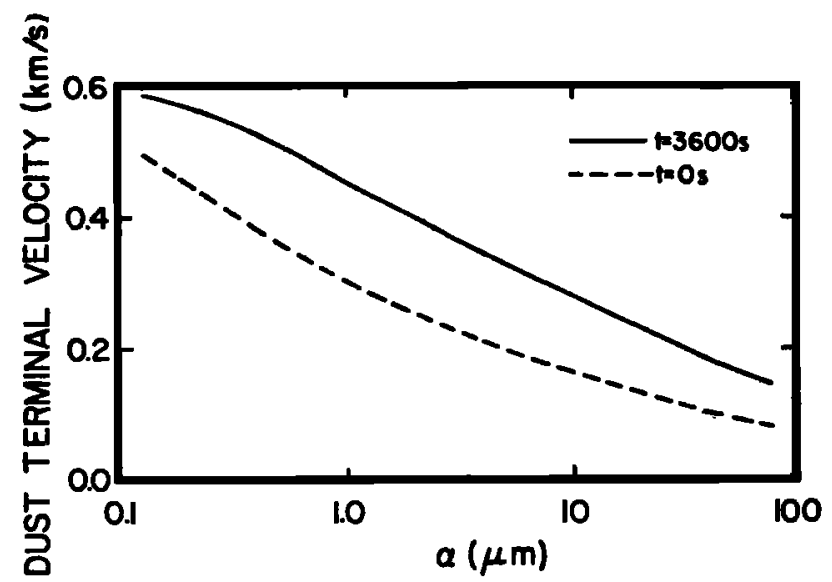

Fig. 1. Dust terminal velocity distributions before and 60 minutes after the onset of the comet outburst. 
DUST SIZE $=0.42 \mu \mathrm{m}$
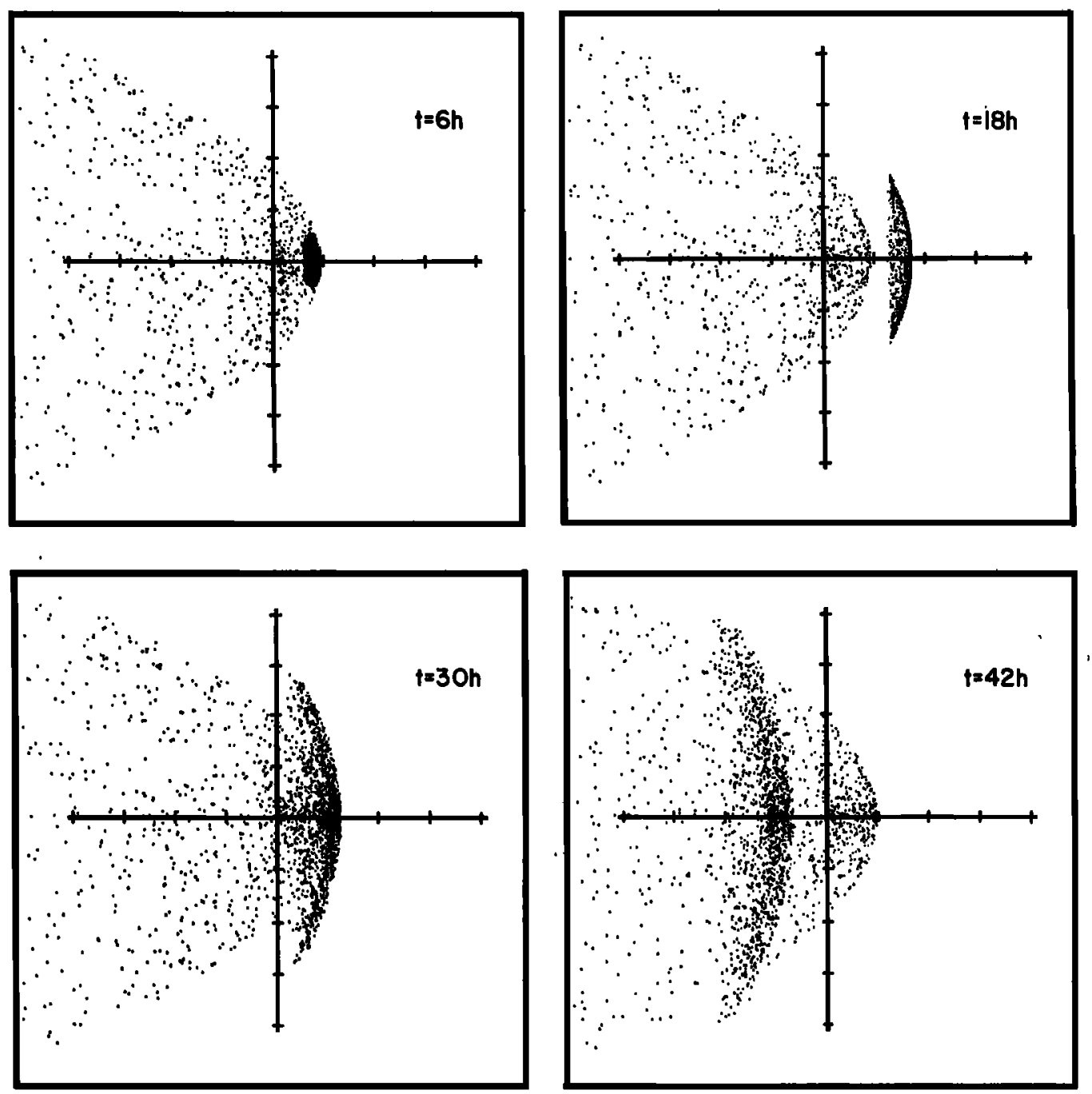

\section{UNIT LENGTH $=10^{4} \mathrm{~km}$}

Fig. 2. Snapshots of $0.42 \mu \mathrm{m}$ dust particle distributions following a $30^{\circ}$ wide subsolar outburst occuring at $\mathrm{t}=0$.

An other important heat source (or sink) is the gas interaction with the infrared radiation field. Recently Marconi and Mendis [1986] published a new approximate expression to describe the combined effect of the Shimizu [1976] - Crovisier [1984] cooling (neutral-neutral collisions rotationally excite the highly polar water molecules which return to ground state by emitting IR radiation) and the gas heating due to infrared radiation trapping. At the surface a Hanner [1983] type dust size distribution (an approximate power law distribution with a sharp cut-off below a critical dust size) was assumed with $a_{0}=0.1 \mu \mathrm{m}, \mathrm{M}=12$, and $\mathrm{N}=4.2$ parameter values.

At a cometocentric distance of $300 \mathrm{~km}$ the dusty gas dynamic treatment was changed for a kinetic method describing the motion of individual dust grains (Horanyi and Mendis [1985]). Beyond this distance the gas-dust interaction can be neglected, and the motion of dust particles are influenced only by solar gravity, radiation pressure and the orbital motion of the comet. It was shown by Horanyi and Mendis [1985] that trajectories of dust particles larger than about $0.5 \mu \mathrm{m}$ are only slightly affected by dust charging; this effect was neglected in our calculations. At the interface the dust velocities were assumed to be radial and time-dependent dust number densities and velocity values were obtained from the dusty gas dynamic calculation.

The present model represents the first comprehensive attempt to calculate the temporal evolution of large scale gas and dust characteristics following a localized comet outburst. At the same time the model has several limitations and shortcomings; most questionable is the assumption of spherical symmetry in the inner coma $(r<300 \mathrm{~km})$. In the next step we intend to improve the model by developing a multidimensional dusty gasdynamic code with improved chemistry and gas-dust interaction model.

\section{Results and discussion}

Following the sudden temperature increase a fast blast wave (not shown) is generated first by the sudden pressure increase at the nucleus. As the blast wave moves through the innermost coma it erodes due to the energy and momentum dissipation 
caused by the gas-dust interaction. Calculated terminal velocity distributions are shown in Figure 1 . It can be seen that the $10 \mathrm{~K}$ surface temperature increase results in larger dust velocities, as well as increased gas and dust production rates. The dust grain velocity increase is localized to a narrow (but slowly expanding) region: this region moves outward like a dust front. The dust density (not shown) exhibits a peak in this transition region, primarily because the particle flux has already jumped to its increased post outburst level, but the velocity has not increased to its new value, yet. The broadening density peak (which might be a factor of 10 higher than the background) is propagating outward in the inner coma. The velocity of this peak is about $0.1-0.2 \mathrm{~km} / \mathrm{s}$, depending on the dust size.

It is interesting to note that the dust terminal velocities are considerable higher during the outburst than before. The increase is about $20 \%$ for the smallest dust grains $(a=0.13 \mu \mathrm{m})$, while large particles $(\sim 100 \mu \mathrm{m})$ exhibit an approximately $100 \%$ velocity increase. According to the Probstein [1968] model of gas-dust interaction, the acceleration of dust particles is proportional to the product of the gas pressure and a function of the gas-dust relative Mach number, consequently the increased gas pressure in the active cometary region results in higher acceleration of dust grains.

Figure 2 shows four spatial distribution snapshots of $0.42 \mu \mathrm{m}$ size dust particles following the subsolar outburst. Six hours after the onset the leading edge of the outburst population has already reached the apex distance of preoutburst particles. At $t=18$ hours the new dust grains reach their apex distance. It is obvious that larger injection velocities result in larger apex distances, because the antisunward force acting on a given size particle needs longer time to turn around an originally faster grain. At $t=18$ hours a gap can be observed between the outburst ejected dust particles and the "old" dust coma. Similar gaps have been observed at various comets, for instance at comet Donati. Later the outburst generated dust population starts to move antisunward under the influance of solar radiation pressure; 30 hours after the outburst the newly formed dust halo and the old coma again overlap. After 42 hours the dust halo has already moved down to the tail and then it slowly diffuses into the background.

\section{Summary}

Evolution of gas and dust distributions following a spatially and temporally localized comet outburst was calculated using a kinetic-hydrodynamic hybrid method. In the inner coma the time-dependent continuity, momentum and energy equations of the dusty gas flow were solved simultaneously, assuming spherical symmetry within the assumed $30^{\circ}$ "jet" and a Propstein [1968] type gas-dust interaction model. In this region a combined photochemical - dust infrared heat source resulted in significant gas heating. In the calculation, 12 dust sizes (logarithmically spaced between 0.1 and $100 \mu \mathrm{m}$ ) were used: dust particles were assumed to follow a Hanner-type size distribution at the surface with a spectral index of -4.2 . Beyond $300 \mathrm{~km}$ a three dimensional kinetic model was used to calculate the trajectory of each individual dust grain. In the dust equation of motion solar radiation pressure, solar gravity and cometary orbital motion were taken into account. At the gas-dust decoupling interface (located at a cometocentric distance of $300 \mathrm{~km}$ ) dust particles were were ejected radially (in the cometocentric frame of refenece) with their size-dependent terminal velocities determined by the dusty gas-dynamic part of the model. The number of injected dust particles was proportional to their number density at the interface surface.

It was found that following the onset of the comet outburst a gas-dust blast wave propagates outward in the inner coma.
About 60 minutes after the increased gas and dust production was initiated at the nucleus a new equilibrium was reached in the inner coma. The most important feature of this new steady state was the significant increase of the dust terminal velocities, due to the increase of gas pressure. These higher terminal velocity values resulted in larger apex distances for dust particles emitted during the outburst. As the dust particles spend a reletively long time near their apex points (the cometocentric velocities are fairly small in this region) therefore the outburst generates a long lasting, distinct dust envelopes in front of the regular dust coma, which later propagates tovard the tail and dissolves. This type of envelope was observed at several comets (cf. comet Donati).

Acknowledgements. This work was supported by NASA grants NAGW-15, NGR-23-005-015, and NSF grants ATM-85-08753 and INT-83-19732 (TIG) and by the Department of Energy under the cooperative contract agreement number DE-FC05-85 ER25000 (HM). Acknowledgement is also made to the National Center for Atmospheric Research, sponsored by the National Science Foundation, and the Supercomputer Computations Research Institute of the Florida State University for computing time used in this research.

\section{References}

Brunner, W., and Michel K.W., Investigation of flow relations in dust - comet comas, Mitt. Astron. Ges., 25, 220, 1968

Crovisier, J., The water molecule in comets: fluorescence mechanisms and thermodynamics of the inner coma, Astron. Astrophys., 130, 361, 1984

Gombosi, T.I., Cravens, T.E., and Nagy, A.F., Time-dependent dusty gasdynamical flow near cometary nuclei, Astrophys. J., 293, 328, 1985

Hanner, M.S., The nature of cometary dust from remote sensing, in Cometary Exploration (Ed. T.I. Gombosi), Hungarian Academy of Sciences, Budapest, Hungary, Vol 2., p1, 1983

Horanyi, M., and Mendis, D.A., Trajectories of charged dust grains in the cometary environment, Astrophys. J.,294, 357,1985

Huebner, W.F., The photochemistry of comets, in The Photochemistry of Atmospheres, (Ed. J.S. Levine), Academic Press, New York, 1985

Kitamura, Y., Axisymmetric dusty gas jet in the inner coma of a comet, Icarus, 1986

Marconi, M.L., and Mendis, D.A., A multi-fluid model of an $\mathrm{H}_{2} \mathrm{O}$-dominated dusty cometary nucleus, The Moon and the Planets, 27, 431,1982

Mendis, D.A., Houpis, H.L.F., and Marconi, M.L., The Physics of Comets, Eundamentals of Cosmic Physics, 10. 1,1985

Orlov, S.V., Komety, ONTI, Moscow, 1935

Probstein, R.F., The dusty gas dynamics of comet heads, in Problems of hydrodynamics and continuum mechanics, published by the Soc. Industr., Appl. Math., p568, 1968

Shul'man, L.M., Hydrodynamics of the circum-nuclear region of a comet, Astromet. Astrofiz., 4, 100, 1969

T. I. Gombosi, Space Physics Research Laboratory, The University of Michigan, Ann Arbor, MI 48109.

M. Horanyi, Supercomputer Computations Research Institute, Florida State University, Tallahassee, FL 32306

(Received January 16, 1986

Accepted January 28, 1986) 